\title{
Experimental Characterization of a Low-Current Cutting Torch
}

\author{
H. Kelly ${ }^{1 *}$ B. Mancinelli ${ }^{2}$, L. Prevosto ${ }^{2}$, F.O. Minotti ${ }^{1 *}$, and A. Márquez ${ }^{1 *}$ \\ ${ }^{1}$ Instituto de Física del Plasma (CONICET), Departamento de Física, \\ Facultad de Ciencias Exactas y Naturales (UBA) Ciudad, Universitaria Pab. I, (1428) Buenos Aires, Argentina \\ ${ }^{2}$ Universidad Tecnológica Nacional, Regional Venado Tuerto, Castelli \\ 501, Venado Tuerto, Pcia. Santa Fe, Argentina
}

Received on 19 December, 2003; revised version received on 28 April, 2004

\begin{abstract}
An experimental characterization of a low-current (30-40 A) cutting torch is presented. To avoid contamination of the plasma arc by removed anode material, a rotating steel cylinder was used as the anode and the arc was anchored onto the cylinder lateral surface. The cathode-anode and cathode-nozzle voltage drops, together with the gas pressure in the plenum chamber were registered for different values of the mass flow rate injected into the plenum chamber. By employing an optical system with a large magnification $(\approx 15 \mathrm{X})$, the arc radius at the nozzle exit was also determined with a digital optical camera. The obtained experimental quantities were used to evaluate several flow properties at the nozzle exit (hot arc plasma and cold gas temperatures, arc and gas velocities, etc.) by employing a simplified theoretical model for the plasma flow in the nozzle. The obtained results are in reasonable agreement with the data reported in the literature by other authors. Explanations of the origin of the clogging effect and the nozzle voltage are also presented.
\end{abstract}

\section{Introduction}

The plasma arc cutting process employs a transferred electric arc between the cathode within a plasma torch and the work piece acting as the anode. A high quality cut requires a high velocity plasma jet. That plasma is created by a narrow constricting nozzle inside the torch, into which the gas-plasma system is injected at a high pressure. The fineness of the nozzle creates a large voltage drop in the plasma along its length, providing intense heating of the plasmagas particles and associated pressure gradient forces, which accelerate the fluids to large velocities[1]. Melting and vaporization of the work piece produces the cut, followed by material displacement along the jet path.

In spite of the widespread application in industry of the plasma arc cutting process, a comprehensive description of this phenomenon has received relatively little research attention. Ramakrishnan et al[2, 3] measured the arc voltage, the cathode-nozzle voltage, the gas pressure at the nozzle's entrance (the so-called plenum chamber) and the diameter of the plasma jet emerging from the nozzle as functions of the torch arc current (in the range 40-160 A). The authors presented also a simplified two-zone fluid model in which all the discharge current was assumed to circulate through a hot central core inside the nozzle, while the remaining structure consisted in a relatively cold neutral gas that thermally insulates the nozzle wall from the arc. Assuming sonic conditions at the nozzle exit and a uniform value for the electric field, integral mass and energy balance equations were stated and solved for the exit pressure, arc radius and nozzle potential. A reasonable good agreement with the experimental results was obtained for an arc temperature of $25000 \mathrm{~K}$ and a gas temperature of $3000 \mathrm{~K}$. Nemchinsky[4] presented also pressure measurements at the plenum chamber for different arc currents (in the range 100-400 A) and compared his results with a more detailed two-zone model to describe the plasma-gas flow in the nozzle. Pardo et al[5] measured the arc voltage as a function of the arc current (in the range 50$180 \mathrm{~A})$ for different nozzle-anode distances and determined radial profiles of the plasma temperature and density from a spectroscopic method (line emission from the arc, assuming an optically thin plasma). Typical plasma temperature obtained in this work at the arc center and immediately below the nozzle exit were from 22000 to $25000 \mathrm{~K}$ for $150 \mathrm{~A}$, from 18000 to $20000 \mathrm{~K}$ for $100 \mathrm{~A}$, and from 14000 to 17000 $\mathrm{K}$ for $50 \mathrm{~A}$. Recently, Freton et al[6] presented an experimental and theoretical study of a plasma cutting torch using, in one experimental configuration, a rotating circular disk anode to whose lateral surface the arc was anchored. From spectroscopic line intensity measurements, an arc temperature of $16500 \mathrm{~K}$ was found on the arc axis at $1 \mathrm{~mm}$ from the nozzle exit for a $30 \mathrm{~A}$ torch.

In this work we present an experimental study of a small cutting torch with a rotating anode operated at arc currents of 30 and $40 \mathrm{~A}$. The cathode-anode and cathode-nozzle voltage drops, together with the gas pressure in the plenum chamber are registered for different values of the mass flow rate injected into the plenum chamber. By employing an optical system with a large magnification $(\approx 15 \mathrm{X})$, the arc radius at the nozzle exit is also determined with a digital optical camera. The obtained experimental quantities are used to evaluate several flow properties at the nozzle exit (hot arc plasma and cold gas temperatures, arc and gas velocities, etc.) by employing a simplified theoretical model for the

${ }^{*}$ Member of the CONICET 
plasma flow in the nozzle [7]. The obtained results are in reasonable agreement with the data reported in the literature by other authors[2, 3, 4, 5, 6]. Explanations of the origin of the clogging effect and the nozzle voltage are also presented.

\section{Experimental arrangement}

The torch used in this study consisted of a cathode centered above an orifice in a straight copper nozzle $(0.8 \mathrm{~mm}$ in diameter). The cathode was made of copper ( $7 \mathrm{~mm}$ in diameter) with a hafnium tip ( $\sim 2 \mathrm{~mm}$ in diameter) inserted at the cathode center. A flow of air cooled the cathode and the nozzle and was also employed as the plasma gas. The gas passed through a swirl ring to provide arc stability. The nozzle consisted in a straight bore $(0.8 \mathrm{~mm}$ in diameter, $2 \mathrm{~mm}$ length) in a copper holder surrounding the cathode (with a separation of $2 \mathrm{~mm}$ ). A scheme of the torch indicating several geometric dimensions is presented in Fig. 1. To avoid plasma contamination by metal vapors from the anode (usually the work piece to be cut), a rotating steel disk with $200 \mathrm{~mm}$ in diameter and $15 \mathrm{~mm}$ thickness was used as the anode. In this study, the anode was located at $8 \mathrm{~mm}$ from the nozzle exit. The arc was transferred to the edge of the disk, and the rotating frequency of the disk was equal to $90 \mathrm{~s}^{-1}$. At this velocity, a well-stabilized arc column was obtained, and practically no metal vapors from the anode were present in the arc.

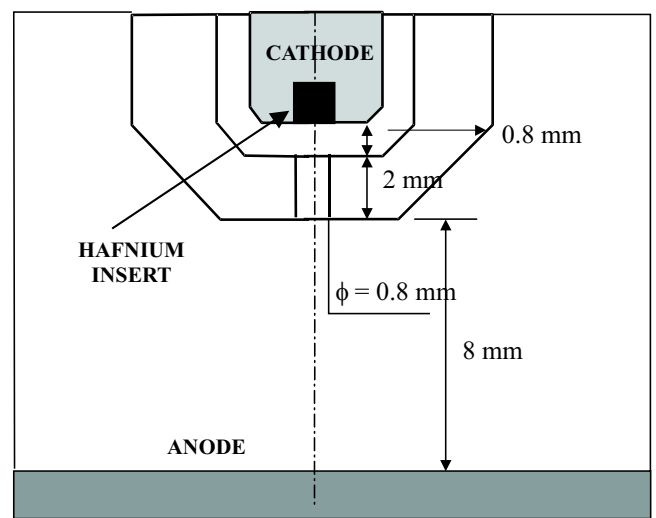

Figure 1. Scheme of the torch indicating several geometric dimensions.

Air was injected into the torch at a nominal pressure of 5 bar, and the pressure in the plenum chamber $\left(p_{N}\right)$ was also measured by connecting a second pressure meter to the cathode-nozzle region through a small hole performed on the lateral surface of the nozzle holder. A mass flow meter was located at the entrance of the torch, but to obtain precise measurements of the mass flow through the nozzle a second mass flow meter was installed at the nozzle exit (with the arc extinguished) in order to take into account eventual gas leakages in the holders of the cathode, swirl ring and nozzle. During arc operation the voltage drop between cathode and anode $\left(\Delta V_{A C}\right)$ and between cathode and nozzle $\left(\Delta V_{N C}\right)$ were registered. By employing a converging lens with 50 $\mathrm{cm}$ of focal length, a highly magnified image $(15 \mathrm{X})$ of the plasma jet at the nozzle exit was obtained on a screen. That image was subsequently registered with a digital camera in order to obtain a measure of the plasma radius at the nozzle exit. In Figs. 2 a sketch of the experimental set up is presented. Fig. 2a) indicates the electrical and mechanical diagnostics, while Fig. 2b) presents a lateral view of the optical diagnostic.

a)

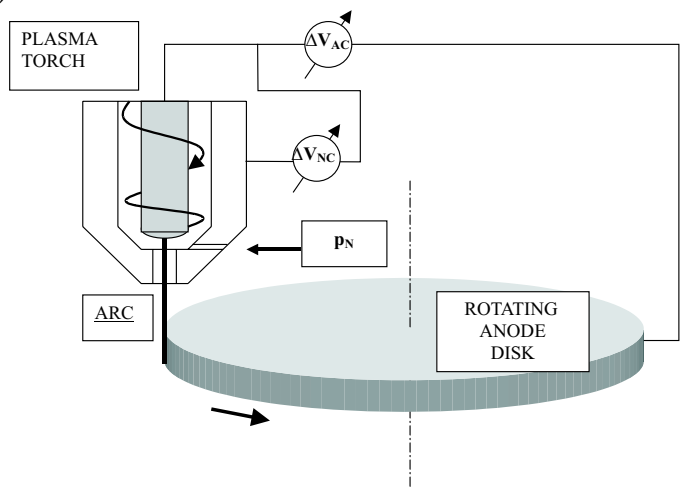

b)

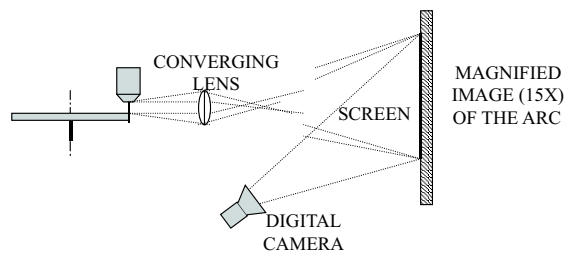

Figure 2. Sketch of the experimental set up. Fig. 2a) indicates the electrical and mechanical diagnostics, while Fig. $2 b$ ) presents a lateral view of the optical diagnostic. $p_{N}$ indicates the gas pressure in the plenum chamber, and $\Delta V_{A C}$ and $\Delta V_{N C}$ the cathode-anode and cathode-nozzle voltage drops, respectively.

The arc was operated at currents $(I)$ with values of 30 and $40 \mathrm{~A}$. For a given $I$ value, the mass flow rate $(\mathrm{dm} / \mathrm{dt})$ through the nozzle was varied in the range $d m / d t=0.06-0.13$ $\mathrm{g} / \mathrm{s}$ for $I=30 \mathrm{~A}$, and $d m / d t=0.05-0.1 \mathrm{~g} / \mathrm{s}$ for $I=40 \mathrm{~A}$.

\section{Results}

In Fig. 3 a qualitative plot of the time evolution of $p_{N}$ is presented. Before arc initiation, $p_{N}$ is stabilized at a certain value $p_{i}$. When the arc is ignited, there is a sudden increase in $p_{N}$ to a value $p_{A}$ (clogging effect), and when the arc is quenched there is also a sudden variation in $p_{N}$ (to a value intermediate between $p_{A}$ and $p_{i}$ ) followed by a slow decrease in $p_{N}$ that tends to the original value $p_{i}$. We have interpreted this behavior as follows: when the arc is ignited the nozzle bore is partially obstructed by the arc, and $p_{N}$ increases due to the combined effects of the obstruction and the heating of the flowing gas in the cathode-nozzle region; when the arc is extinguished, the nozzle bore is suddenly removed from the obstruction but the gas remains hot, so there is an abrupt decrease of $p_{N}$ from $p_{A}$ to $p_{i}$ followed of a gradual decrease of $p_{N}$ as the gas is progressively cooled. 
Within the frame of this interpretation, the pressure drop $p_{A}-\Delta p-p_{i}$ measured at the moment the arc is quenched (see Fig. 3) is a measure of the gas heating in the cathodenozzle region during the discharge.

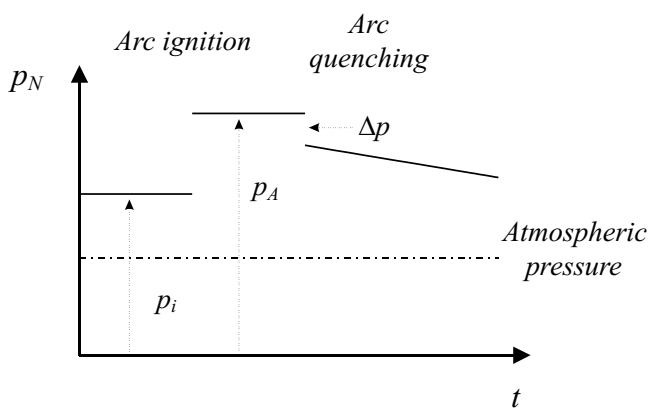

Figure 3. Qualitative plot of the time evolution of the plenum chamber pressure $\left(p_{N}\right)$.

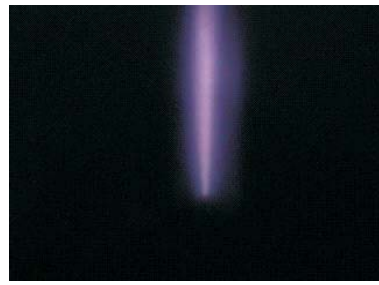

a)

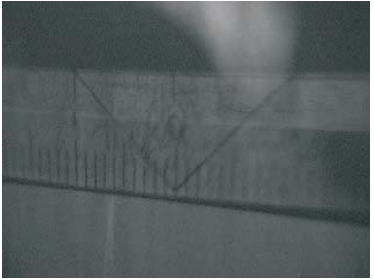

b)
Figure 4. Fig. 4a) Image of the arc registered by the optical system. Since the lens invert the image, the nozzle exit corresponds to the bottom of the figure, while the lateral surface of the rotating disk is located at the top. Fig. 4b) Image of a graduate scale located at the same arc position.

In Fig. 4a) an image of the arc registered by the previously described optical system is presented. Since the lens invert the image, the nozzle exit corresponds to the bottom of the figure, while the lateral surface of the rotating disk is located at the top. Fig. 4b) shows an image of a graduate scale located at the same arc position, which was employed to calibrate the size of the arc images. The arc emerges from the nozzle with a radius $r_{A}=(0.3 \pm 0.1) \mathrm{mm}$, independent of the investigated values of $I$ and $d m / d t$. The relatively large uncertainty in the $r_{A}$ values is due to the pixel's size of the digital camera together with some difficulty in the determination of the arc edge.

In Table I the results of the measurements of $p_{i}, p_{A}, \Delta p$, $p_{A}-\Delta p-p_{i}, \Delta V_{A C}$, and $\Delta V_{N C}$ as functions of $d m / d t$ and for $I=30 \mathrm{~A}$ and $40 \mathrm{~A}$ are presented.

It can be seen from Table I that, as expected, $p_{i}$ increases with $d m / d t$, and correspondingly $p_{A}$ presents the same behavior with $d m / d t$. However, $\Delta p$, which accordingly to the interpretation previously pointed out is related to the obstruction of the nozzle's bore by the arc, presents also an increasing behavior with $d m / d t$, that can not be explained in terms of variations of $r_{A}\left(r_{A} \approx\right.$ const.). We will show in the next section that the variations in $\Delta p$ can be explained in terms of changes in the values of $d m / d t$ when the arc is in operation. It is worth noting that the measured values of $d m / d t$ in Table I correspond to a situation in which the arc is extinguished, so when the arc is operated (and partially obstruct the nozzle's bore) the true value of $\left.d m / d t(d m / d t)_{A}\right)$ can be somewhat changed.

It can also be seen from Table I that the arc voltage drop $\Delta V_{A C}$ reaches relatively high values, consistent with the large cathode-anode gap $(\approx 11 \mathrm{~mm})$ settled in this experiment. $\Delta V_{A C}$ also present relatively small variations with $d m / d t$, which can be explained in terms of small variations in $r_{A}$ (smaller than the experimental uncertainty of $r_{A}$, because $\Delta V_{A C}$ is quite sensitive to changes in $r_{A}, \Delta V_{A C} \sim r_{A}^{-2}$ ) or variations in the arc temperature. The cathode-nozzle voltage drop also presents an increasing behavior with $\mathrm{dm} / \mathrm{dt}$ (similar to the behavior of $\Delta V_{A C}$ ), and an explanation of these values will be given in the next Section by using the Quick Field electromagnetic program[8].

\section{Discussion}

In order to interpret the experimental results presented in the previous section, some of them were compared with theoretical results obtained from a simplified hydrodynamic model for the arc in the torch nozzle[7]. That model uses a two-zone approximation (a hot central plasma carrying the discharge current surrounded by a relatively cold gas that thermally isolates the nozzle wall from the plasma) and allows to obtain the profiles of the physical quantities along the nozzle in terms of the externally controlled parameters of the torch (geometry of the torch, discharge current, mass flow of gas and plenum pressure) and the values of the arc and gas temperatures at the nozzle entrance.

In our experimental situation, the gas temperature $\left(T_{G}\right)$ at the nozzle entrance $(x=0)$ was determined from the pressure increase, $p_{A}-\Delta p-p_{i}$, which as discussed previously, is associated to the gas heating. It results $T_{G}(x=0)=370$ $\mathrm{K}$ (that is, $70 \mathrm{~K}$ over the ambient temperature). The arc temperature $\left(T_{A}\right)$ at the nozzle entrance was employed as a parameter of the problem and was finally chosen so as to obtain values of the arc temperatures at the nozzle exit similar to those reported in the literature $[5,6]$ for similar operating current-values torches.

For the determination of the mass flow of gas actually flowing along the nozzle during arc operation, it must be remarked that the values presented in Table I correspond to measurements performed with a mass flow meter located at the nozzle exit with the arc extinguished. Since in practice during arc operation the hot plasma flowing along the nozzle carries a negligible mass as compared with the mass flow involved in the surrounding cold gas, the presence of the arc leads to a partial obstruction of the nozzle's bore, which in turn can change the actual value of mass flow of gas $\left.(d m / d t)_{A}\right)$ with respect to that registered without $\operatorname{arc}(d m / d t)$. To take into account this effect, we have employed the global conservation laws of mass, momentum, and energy (including a partial obstruction of the nozzle's bore, and the energy transferred from the arc to the cold gas) to relate $d m / d t)_{A}$ with $d m / d t$ for known values of the gas parameters at the nozzle entrance. Using the actual values of the gas parameters in the experiment the obtained relation predicts $d m / d t)_{A} \approx 0.5 d m / d t$. 
TABLE I: Measurements of plenum chamber pressure without arc $\left(p_{i}\right)$, plenum chamber pressure with arc $\left(p_{A}\right)$, change in the pressure due to nozzle bore obstruction ( $\Delta p)$, change in the pressure due to gas heating $\left(p_{A}-\Delta p-p_{i}\right)$, arc voltage drop $\left(\Delta V_{A C}\right)$, and cathode-nozzle voltage drop ( $\left.\Delta V_{N C}\right)$ as functions of the gas mass flow rate $(d m / d t)$ and for $I=30 \mathrm{~A}$ and $40 \mathrm{~A}$.

\begin{tabular}{|c|c|c|c|c|c|c|c|}
\hline $\begin{array}{c}I \\
(\mathrm{~A})\end{array}$ & $\begin{array}{c}d m / d t \\
(\mathrm{~g} / \mathrm{s})\end{array}$ & $\begin{array}{c}p_{i} \\
(\mathrm{bar})\end{array}$ & $\begin{array}{c}p_{A} \\
(\text { bar })\end{array}$ & $\begin{array}{c}\Delta p \\
(\mathrm{bar})\end{array}$ & $\begin{array}{c}p_{A}-\Delta p-p_{i} \\
(\mathrm{bar})\end{array}$ & $\begin{array}{c}\Delta V_{A C} \\
(\mathrm{~V})\end{array}$ & $\begin{array}{c}\Delta V_{N C} \\
(\mathrm{~V})\end{array}$ \\
\hline 30 & $0.057 \pm 0.005$ & $1.05 \pm 0.05$ & $1.5 \pm 0.05$ & $0.20 \pm 0.1$ & $0.20 \pm 0.1$ & $125 \pm 5$ & $21 \pm 3$ \\
\hline & $0.077 \pm 0.005$ & $1.1 \pm 0.05$ & $1.7 \pm 0.05$ & $0.4 \pm 0.1$ & $0.20 \pm 0.1$ & $135 \pm 5$ & $22 \pm 3$ \\
\hline & $0.096 \pm 0.005$ & $1.25 \pm 0.05$ & $1.8 \pm 0.05$ & $0.4 \pm 0.1$ & $0.20 \pm 0.1$ & $135 \pm 5$ & $23 \pm 3$ \\
\hline & $0.130 \pm 0.005$ & $1.3 \pm 0.05$ & $2.15 \pm 0.05$ & $0.7 \pm 0.1$ & $0.20 \pm 0.1$ & $145 \pm 5$ & $30 \pm 3$ \\
\hline 40 & $0.052 \pm 0.005$ & $1.05 \pm 0.05$ & $1.45 \pm 0.05$ & $0.25 \pm 0.1$ & $0.20 \pm 0.1$ & $124 \pm 5$ & $18 \pm 3$ \\
\hline & $0.077 \pm 0.005$ & $1.1 \pm 0.05$ & $1.75 \pm 0.05$ & $0.4 \pm 0.1$ & $0.20 \pm 0.1$ & $135 \pm 5$ & $22 \pm 3$ \\
\hline & $0.104 \pm 0.005$ & $1.25 \pm 0.05$ & $2.2 \pm 0.05$ & $0.8 \pm 0.1$ & $0.20 \pm 0.1$ & $144 \pm 5$ & $27 \pm 3$ \\
\hline
\end{tabular}

TABLE II: Theoretical results obtained from the model. In all the cases $T_{A}(x=0)=10000 \mathrm{~K}$, and $x=L$ indicates the nozzle exit. The arc radius at the nozzle entrance was selected so as to reach an almost constant arc radius at the nozzle exit of $0.32 \mathrm{~mm}$ (in accordance with the measured value), and the theoretical solution corresponds to a sonic point of the arc at the nozzle exit. The quantity $\left.=d m / d t)_{G} / d m / d t\right)_{A}$ indicates the mass flow fraction carried by the gas, $R_{A}$ indicates the arc radius, $u_{A}$ and $u_{G}$ the arc and gas velocities and $\Delta V$ the voltage drop along the nozzle.

\begin{tabular}{|c|c|c|c|c|c|c|c|c|c|c|c|}
\hline $\begin{array}{c}\mathrm{I} \\
(\mathrm{A})\end{array}$ & $\begin{array}{c}p_{A}(0) \\
(\mathrm{bar})\end{array}$ & $\begin{array}{c}d m / d t)_{A} \\
(\mathrm{~g} / \mathrm{s})\end{array}$ & $\xi$ & $\begin{array}{c}R_{A}(0) \\
(\mathrm{mm})\end{array}$ & $\begin{array}{c}R_{A}(L) \\
(\mathrm{mm})\end{array}$ & $\begin{array}{c}u_{A}(L) \\
(\mathrm{m} / \mathrm{s})\end{array}$ & $\begin{array}{c}u_{G}(L) \\
(\mathrm{m} / \mathrm{s})\end{array}$ & $\begin{array}{c}T_{A}(L) \\
(\mathrm{K})\end{array}$ & $\begin{array}{c}T_{G}(L) \\
(\mathrm{K})\end{array}$ & $\begin{array}{c}p_{A}(L) \\
(\mathrm{bar})\end{array}$ & $\begin{array}{c}\Delta V \\
(\mathrm{~V})\end{array}$ \\
\hline 30 & 1.50 & 0.029 & 0.80 & 0.38 & 0.32 & 3700 & 670 & 18800 & 1680 & 0.98 & 21 \\
\hline & 1.70 & 0.038 & 0.83 & 0.37 & 0.32 & 3400 & 610 & 18300 & 1360 & 1.13 & 22 \\
\hline & 1.80 & 0.050 & 0.87 & 0.37 & 0.31 & 3300 & 560 & 18400 & 1070 & 1.22 & 22 \\
\hline & 2.15 & 0.070 & 0.90 & 0.36 & 0.31 & 2400 & 460 & 18200 & 870 & 1.75 & 22 \\
\hline 40 & 1.45 & 0.026 & 0.83 & 0.38 & 0.32 & 5500 & 830 & 26300 & 1950 & 0.80 & 22 \\
\hline & 1.75 & 0.038 & 0.85 & 0.38 & 0.32 & 5000 & 740 & 23000 & 1400 & 1.00 & 25 \\
\hline & 2.20 & 0.055 & 0.85 & 0.37 & 0.32 & 4400 & 650 & 19800 & 1100 & 1.20 & 27 \\
\hline
\end{tabular}

In Table II the theoretical results obtained from the model are presented. In all the cases $T_{A}(x=0)=10000 \mathrm{~K}$, and $x=L$ indicates the nozzle exit. The arc radius at the nozzle entrance was selected so as to reach an almost constant arc radius at the nozzle exit of $0.32 \mathrm{~mm}$ (in accordance with the measured value), and the theoretical solution corresponds to a sonic point of the arc close to the nozzle exit. The quantity $\left.\xi=d m / d t)_{G} / d m / d t\right)_{A}$ indicates the mass flow fraction carried by the gas, $R_{A}$ indicates the arc radius, $u_{A}$ and $u_{G}$ the arc and gas velocities and $\Delta V$ the voltage drop along the nozzle.

From Table II, it can be seen that the neutral gas carries the main part of the mass flow (between 80 and $90 \%$ ). The arc velocities are in the range of several thousands of $\mathrm{m} / \mathrm{s}$, while the gas velocities are in the range of several hundreds of $\mathrm{m} / \mathrm{s}$ (both velocities present a decreasing behavior with $d m / d t)_{A}$ ). The arc temperatures at the nozzle exit reach values in the range 18000-26000 K (increasing with the arc current), while the gas temperature is in the range 800 $2000 \mathrm{~K}$ (increasing with $I$ and decreasing with $d m / d t)_{A}$ ). The pressure at the nozzle exit is close to 1 Atmosphere, excepting the case were a large mass flow is injected into the nozzle (fourth row in the Table) thus producing a high pressure value at the nozzle exit. In this case, the emerging flow matches the atmospheric pressure value by a rarefaction wave[5]. The nozzle voltage drop $\Delta V$ ranges from 21 to $27 \mathrm{~V}$, and the product $\Delta V I$ represent the Joule power delivered into the nozzle.

In order to predict the cathode-nozzle voltage and compare this prediction with the measured values, we use the Quick Field program[8]. Quick Field is a finite element analysis package for electromagnetic simulation. In this case, lines of constant electrostatic potential can be obtained for a region with prescribed boundary conditions. In Fig. 5 a typical output of the Quick Field program is presented, for the case $I=30 \mathrm{~A}, d m / d t)_{A}=0.05 \mathrm{~g} / \mathrm{s}, \Delta V_{A C}=135$ $\mathrm{V}$. In this simulation, the cathode and anode voltages were set at -135 and $0 \mathrm{~V}$, respectively; and the arc radius was supposed to vary linearly inside and outside the nozzle. The arc temperature was: $T_{A}=18000 \mathrm{~K}$ in the cathode-nozzle region, $T_{A}=16000 \mathrm{~K}$ inside the nozzle, and $T_{A}=10000 \mathrm{~K}$ in the nozzle-anode region. The arc electrical conductivity was calculated according the quoted temperatures in each region. The resulting nozzle voltage is $14 \mathrm{~V}$, which corresponds to the measured voltage in this case $(23 \mathrm{~V})$ if a cathode sheath voltage drop of $\sim 10 \mathrm{~V}$ is assumed[6]. This last value 
is characteristic for high-pressure arcs, where the cathode electron emission mechanism is mainly thermo ionic, therefore requiring a relatively small sheath voltage drop to provide the discharge electrons[9]. Quick Field calculations for other cases gave similar agreement with the experimental cathode-nozzle voltage.
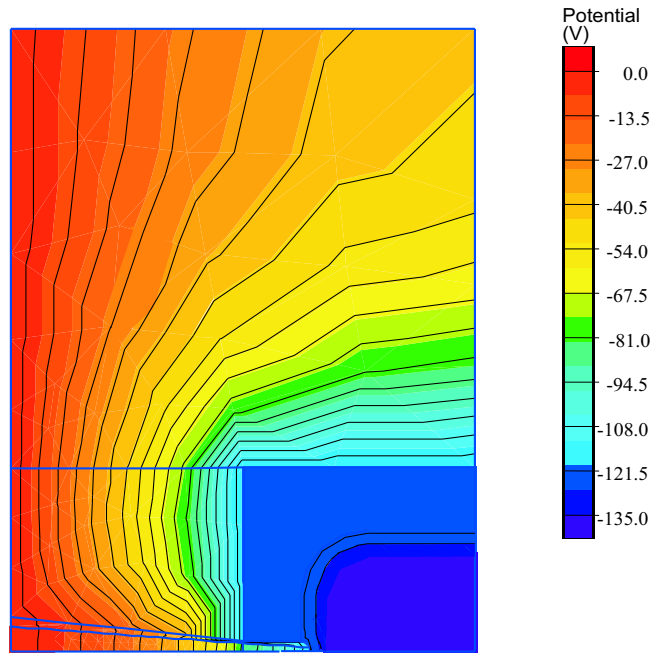

Figure 5. Lines of constant electrostatic potential obtained from the Quick Field program for the case $I=30 \mathrm{~A}, d m / d t)_{A}=0.05 \mathrm{~g} / \mathrm{s}$, $\Delta V_{A C}=135 \mathrm{~V}$. Only half of the device is shown, the lower boundary of the figure corresponding to the axis of the system. The block at $-135 \mathrm{~V}$ in the lower right side represents the cathode. The equipotential block, of rectangular external boundary, around the cathode represents the nozzle. The anode, at $0 \mathrm{~V}$, corresponds to the left boundary. The arc runs along the axis (lower boundary) between cathode and anode.

\section{Final remarks}

An experimental characterization of a low-current (30-40 A) cutting torch has been presented. To avoid contamination of the plasma arc by removed anode material, a rotating steel cylinder was used as the anode and the arc was anchored onto the cylinder lateral surface.

The cathode-anode and cathode-nozzle voltage drops, together with the gas pressure in the plenum chamber were registered for different values of the mass flow rate injected into the plenum chamber. By employing an optical system with a large magnification $(\approx 15 \mathrm{X})$, the arc radius at the nozzle exit was also determined with a digital optical camera. The time evolution of the plenum chamber pressure indicates that the clogging effect can be separated into two comparable contributions; one of them is due to the nozzle bore obstruction by the presence of the arc, while the other is due to gas heating in the plenum chamber.

The results of this experiment show that care must be taken for the determination of the actual gas mass flow rate, either by taking into account the gas leakage in the torch when that mass flow is measured with the arc operating, or by evaluating the effect of the nozzle bore obstruction on the flow rate when this quantity is measured with the arc extinguished.

The obtained experimental quantities were used to evaluate several flow properties at the nozzle exit (hot arc plasma and cold gas temperatures, arc and gas velocities, etc.) by employing a simplified theoretical model for the plasma flow in the nozzle. The obtained results are in reasonable agreement with the data trend reported in this experiment or with several data reported in the literature by other authors.

\section{Acknowledgements}

This work was supported by a grant from the Universidad de Buenos Aires (PID X214).

\section{References}

[1] R. C. Fernicola, Weld. J. 77, 52 (1998).

[2] S. Ramakrishnan, M. Gershenzon, F. Polivka, T. N. Kearny, and M. W. Rogozinsky, IEEE Trans. Plasma Sci. 25, 937 (1997).

[3] S. Ramakrishnan and M. W. Rogozinsky, J. Phys. D: Appl. Phys. 30, 636 (1997).

[4] V. A. Nemchinsky, J. Phys. D: Appl. Phys. 31, 3102 (1998).

[5] C. Pardo, J. González-Aguilar, A. Rodríguez-Yunta, and M. A. G. Calderón, J. Phys. D: Appl. Phys. 32, 2181 (1999).

[6] P. Freton, J. J. Gonzalez, A. Gleizes, F. Camy Peyret, G. Caillibotte, and M. Delzenne, J. Phys. D: Appl. Phys. 35, 115 (2002).

[7] H. Kelly, F. O. Minotti, L. Prevosto, and B. Mancinelli, "Hydrodynamic model for the plasma gas flow in a cutting torch nozzle", submitted to Brazilian Journal of Physics, 2003.

[8] http://www.quickfield.com

[9] Y. P. Raizer, Gas Discharge Physics, Springer-Verlag, Berlin, 1991. 